\title{
sciendo
}

\author{
BULGARIAN ACADEMY OF SCIENCES
}

CYBERNETICS AND INFORMATION TECHNOLOGIES • Volume 21, No 1

Sofia $\bullet 2021 \quad$ Print ISSN: 1311-9702; Online ISSN: 1314-4081

DOI: $10.2478 /$ cait-2021-0011

\section{Systems Engineering Information Model of Vehicle-Pedestrian Collisions}

\author{
Hristo Uzunov, Plamen Matzinski, Silvia Dechkova, Nikolay Dimov \\ Technical University of Sofia, Faculty and College - Sliven, Bulgaria \\ E-mails: hvuzunov@gmail.com matzinskipl@tu-sofia.bgrsi_yana@abv.bgnndimov78@yahoo.com
}

Abstract: Engineering analysis of motor vehicle collisions as a complex type of research combines the application of scientific approaches from different fields: mechanics, mathematics, structural design, etc. This implies accurate and unambiguous determination of input data and their application in computational procedures for finding solutions in iterative mode. Hence, the reason to apply the specific research method of information modelling in the present study to one of the main types of road accidents, namely the pedestrian-car collision.

Keywords: Automobile, pedestrian, road traffic accident, information model, SolidWorks.

\section{Introduction}

Traffic collision reconstruction is the best example of engineering analysis involving processes of analyzing and interpreting complex data. The accuracy of the obtained intermediate and final results, respectively, of the calculation procedures play a crucial role in the analysis and identification of causes and factors in road accidents. Engineering analysis applied to the investigation of road traffic accidents influences judicial decision-making. The conclusions, determining measures for accident prevention, are also essential for the public. The above statements presuppose the demand to apply the well-known in natural science and engineering Systems Approach [1,5]. The systematic approach implies that the designer has a conceptual model seeking to represent empirical objects - mathematical model, geometric model, system information model.

Expertise research on road accidents meets complex criteria determined by modelling physical processes describing the problem area. The high requirements for accuracy in calculating the velocity of the center of mass of all participants in the road traffic accident at the time of the impact as well as pre-impact and post-impact velocities are essential for the concept creation and the proposed technical solution $[2,3,14-16,19]$. 
Automotive engineers work with various types of models, the basic ones being information, mathematical and geometric models. They are closely related to each other and together determine the properties of the real world objects, recreated in models (prototypes). Information modelling, in turn, connects the description of a real object with specific data. In such cases it is essential to define the necessary and sufficient data to be accurately and successfully implemented. On the other hand, information modelling is closely connected with mathematical modelling, where the properties and behaviour of the object are apparent and described in order to solve dependency related issues [4, 17]. Geometric modelling is an integral part of information and mathematic modelling when studying material objects. In these cases, mathematical modelling also provides the empirical examination and structural dependencies, to varying degrees, necessary to geometrically construct the prototype of the object. This is usually associated with the representation of a complex real object, by connecting separate geometric primitives (sphere, cylinder, cone, prism, etc.). This, together with the means of descriptive geometry, is the basis for solving engineering problems related to the geometric reproduction of images of technical objects. The role of this type of modelling in automotive engineering tasks is also associated with the processes of motion simulation of the real object prototype. The generated geometric models can be two-dimensional (2D) or three-dimensional (3D).

Taking into consideration the abovementioned it can be pointed out that the basic types of modelling are closely related to each other. Their analysis and description should be based on the specific characteristics of the interconnectedness among them as well.

Automation of engineering tasks and optimization of their solutions respectively is unthinkable without stored data based on previous research and case studies as well as accumulated expertise knowledge about their application. Thus, a large body of engineering and practical experience is compiled to create preconditions for quick elimination of impossible or irrational solutions.

The systems engineering information model proposed in this paper aims to raise awareness about the automation of information processes that could be implemented in investigation and traffic collision reconstruction as an integral part of road traffic accident research between pedestrians and motor vehicles.

The basis of any modelling is the sound field data and the conceptual model of their interpretation. Fig. 1 represents systems engineering information model of a vehicle-pedestrian collision.

The information model has a predominant role in the present study. The gathered input data from the accident investigation and their interpretation are a prerequisite for the design of geometric and mathematical models. The geometric model is decisive for the reconstruction of motion of traffic participants in road accidents. On the other hand, the description of motion represents data-driven analysis of dependencies based on analytic methodology. Therefore, the geometric and mathematical models are in complex interconnection and their artificial division and separate determination would not be correct. As a result, each of the abovementioned three types of models have been considered with its peculiarities in the present study. 


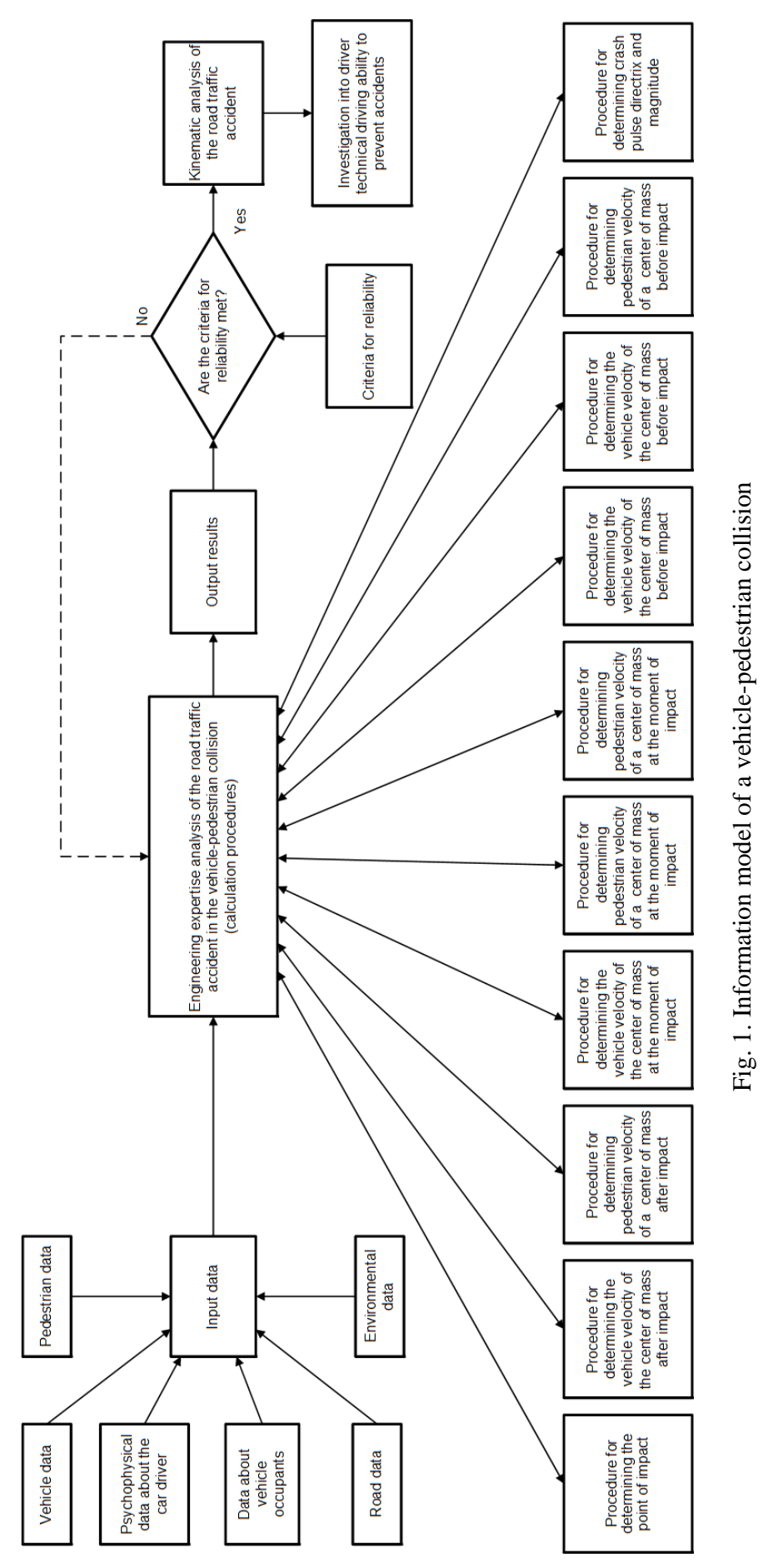




\section{Creating geometric models of vehicle-pedestrian collisions in SolidWorks software environment}

Free shapes of surfaces are used to describe the silhouette of a 3D geometric element. The shapes of freeform surfaces and spatial curves are stored in CAD software by their poles, curvature and a number of patches. The degree of a surface is determined by its mathematical properties and represents the surface as a polynomial with variable gradients and values. The order of the polynomial that describes the surface is one greater than the degree, and is equal to the number of coefficients, not the greatest degree of the surface.

Surface patches are merged into a single surface by knot lines (intersections). The number of knots determines the influence of the poles on the two merged surfaces as well as the smoothness of the transition.

\subsection{Geometric car model}

The car model is assembled from several separate parts with the corresponding movable and immovable connections between them. For each detail, its geometric dimensions and material they are made from are set. Based on these input data, CAD system automatically determines the mass parameters of the object.

In accordance with the selected generalized coordinates for dynamic research, the multi-mass car model is structured in two modules - a body (Fig. 2) and a running gear (Fig. 3).

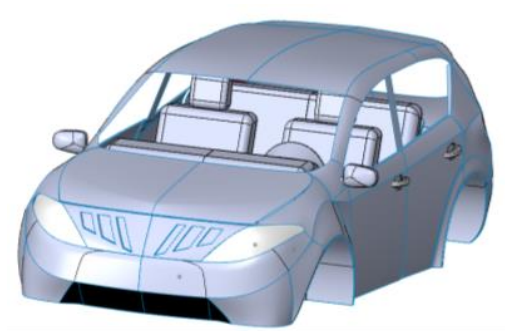

Fig. 2. Car model representing unsprung mass

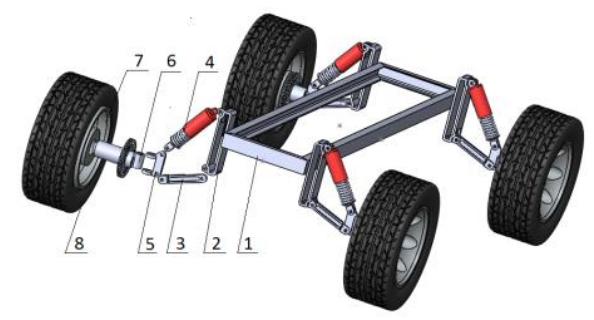

Fig. 3. Car suspension

Thus, the unsprang mass is represented in a fully-assembled unit (assembly) including the elements of the body, equipment and engine. The sprung mass is composed of the wheels and their suspension attached to the frame. The connections between the separate elements are movable and immovable depending on the degrees of freedom.

\subsection{Geometric model of a pedestrian}

The 3D model is built with the help of pre-defined objects - rectangular prisms, cylinders, spheres, etc. Boolean operations are employed to unite, divide and subtract features to define complex details.

The created bodies are connected by articulations, and the human gait completely corresponds to the different types of walking behaviour - slow, fast, 
running and fast running. For this purpose, restrictions are introduced in the generalized coordinates of the angular rotations at the limbs.

The person consists of the following elements: 1 - head; 2 - joint; 3 -torso; 4 - upper limb; 5 - lower limb (Fig. 4a).

The spatial model of a human body is a multimass system of $n_{i}(i=1, \ldots, 26)$ number of bodies connected by articulations and have their own local Cartesian coordinate system $C_{i} x_{i}^{\prime \prime} y_{i}^{\prime \prime} z_{i}^{\prime \prime}$ relative to the movable $C x^{\prime} y^{\prime} z^{\prime}$. Each body has its own law of relative motion with respect to the movable coordinate system (Fig. 4b). In each joint there are restrictions at specified angle of rotation.

The absolute motion of the body is described by the law of motion. When the person is a pedestrian, four phases of a car impact are valid. Phase 1 - boundaryvalue problem of initial impact; Phase 2 - throw distance and repeated landings during the sliding phase on the car front; Phase 3 - falling body until its resting position before it hits the contact surface; Phase 4 - rolling body and its sliding along the contact surface. When a person is a passenger or an occupant in a car, their movement is restricted by the interior equipment of the car.

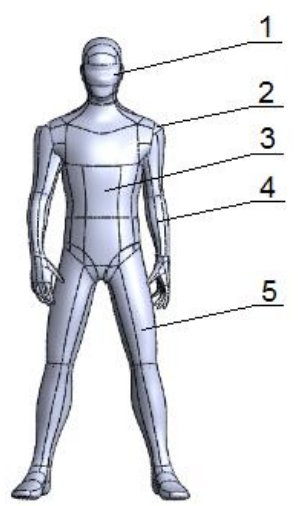

(a)

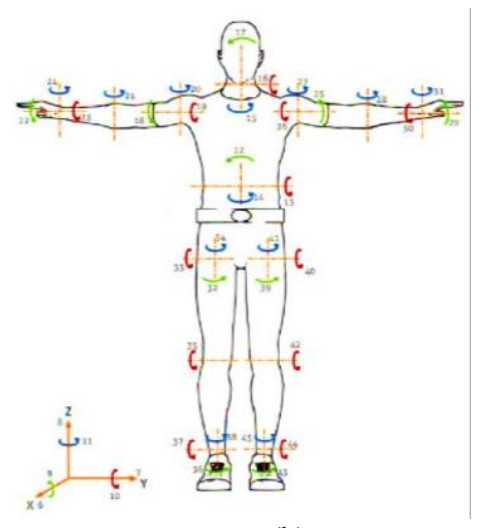

(b)

Fig. 4. Human 3D model created in SolidWorks

\subsection{Geometric road model}

The road is modelled as a plane positioned in the plane of the $X$ and $Y$ axes of a fixed coordinate system. The longitudinal $X$-axis coincides with the dividing line of the road lanes. The length of the road section is chosen from the location of the accident area.

\subsection{Multi-body mechanical system}

Fig. 5 shows the spatial models of three objects, namely a road, an automobile and a human body. Fixed $X, Y, Z$ coordinate system is specified. A permanently connected coordinate system $x^{\prime}, y^{\prime}, z^{\prime}$ is introduced and it is permanently connected to the separate elements $-x_{i}^{\prime \prime}, y_{i}^{\prime \prime}, z_{i}^{\prime \prime}(i=1, \ldots, n$, and $n$ is the number of moving body elements). 

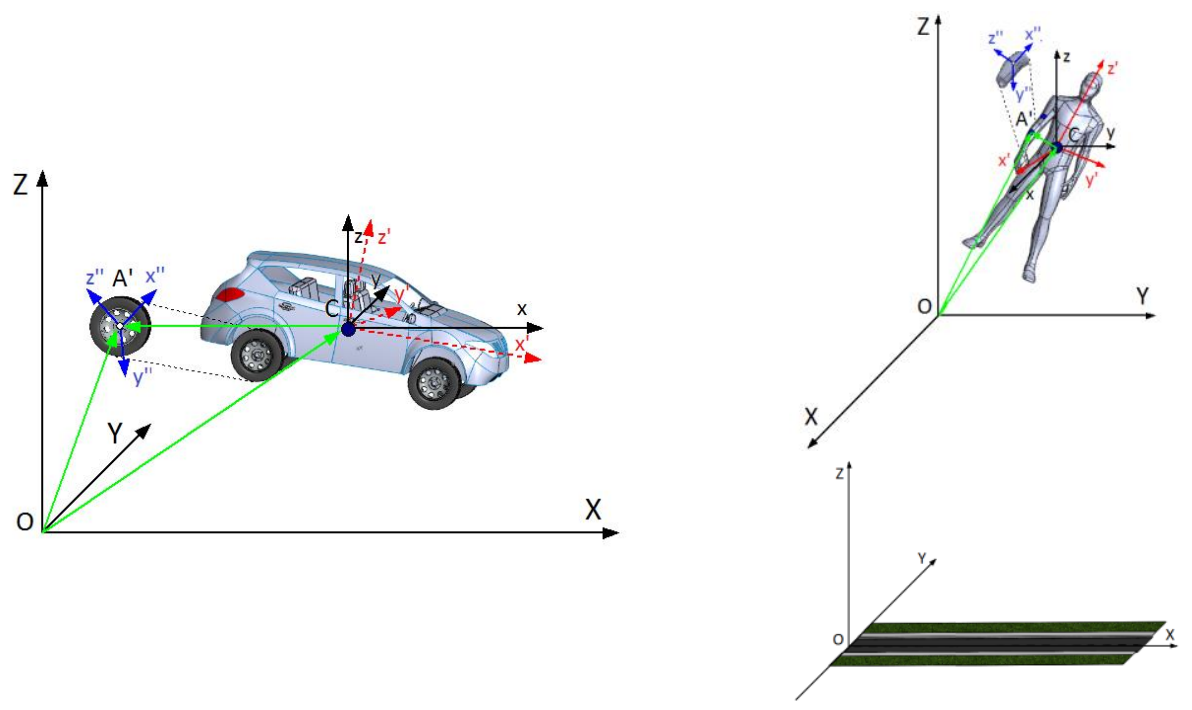

Fig. 5. Spatial models

The radius vector $O C$ determines the position of the center of mass of the object in the $X, Y, Z$ coordinate system. The position of each composite element is determined by six generalized coordinates, three rotations and three translations. The transition from one coordinate system to another is performed by means of the following vector:

$$
\overrightarrow{O A}=\overrightarrow{O C}+\overrightarrow{C A}
$$

The coordinates of an arbitrary point of an element are determined by

$$
\left|\begin{array}{l}
x_{A_{i}} \\
y_{A_{i}} \\
z_{A_{i}}
\end{array}\right|=\left|\begin{array}{l}
X_{C} \\
Y_{C} \\
Z_{C}
\end{array}\right|+\left|\begin{array}{lll}
a_{11} & a_{12} & a_{13} \\
a_{21} & a_{22} & a_{23} \\
a_{31} & a_{32} & a_{33}
\end{array}\right| \cdot\left|\begin{array}{c}
x_{A}^{\prime} \\
y_{A}^{\prime} \\
z_{A}^{\prime}
\end{array}\right| .
$$

The created 3D models are positioned on a chosen fixed Cartesian coordinate system with a common origin of the coordinates $x_{C_{i}}$ and $y_{C_{i}}$. The $z_{C_{i}}$ coordinate is chosen according to the geometric arrangement of the center of mass of the given object.

\section{Transformation and transfer of the created complex geometric model of pedestrian and automobile in Matlab-Simulink environment}

Fig. 6 represents a block diagram outlining the export of the created complex model of Pedestrian and Automobile to Matlab-Simulink environment.

The geometric models created in Solidworks environment are transferred into Matlab-Simulink environment applying Matlab-Simulink software application to achieve correct reconstruction of motion of the objects in the road traffic accident. These models of the participants, part of the so-called complex model, are of utmost importance for vehicle crashes. 


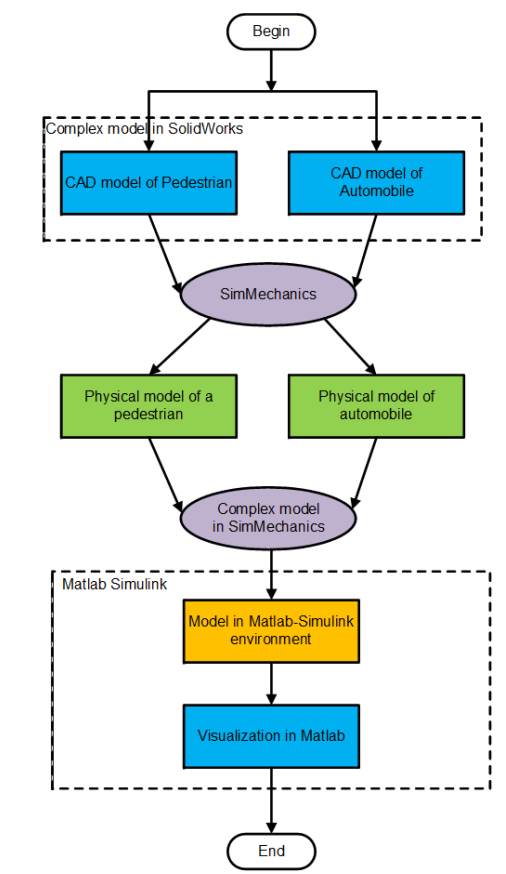

Fig. 6. Block-diagram of the export of the complex model of Pedestrian and Automobile to Matlab-Simulink environment

A control model has been created using Matlab-Simulink software according to kinematic laws of uniform, accelerated and decelerated motion in mechanics. The object of control has been the common center of mass for the system. In addition, the knee joints and ankle joints of the upper and lower limbs has been controlled by sinusoidal law and rotational dephasing.

Fig. 7 shows the control zone in Cartesian coordinates of the center of mass of the system, as well as the angular rotation about the vertical $O z$-axis.
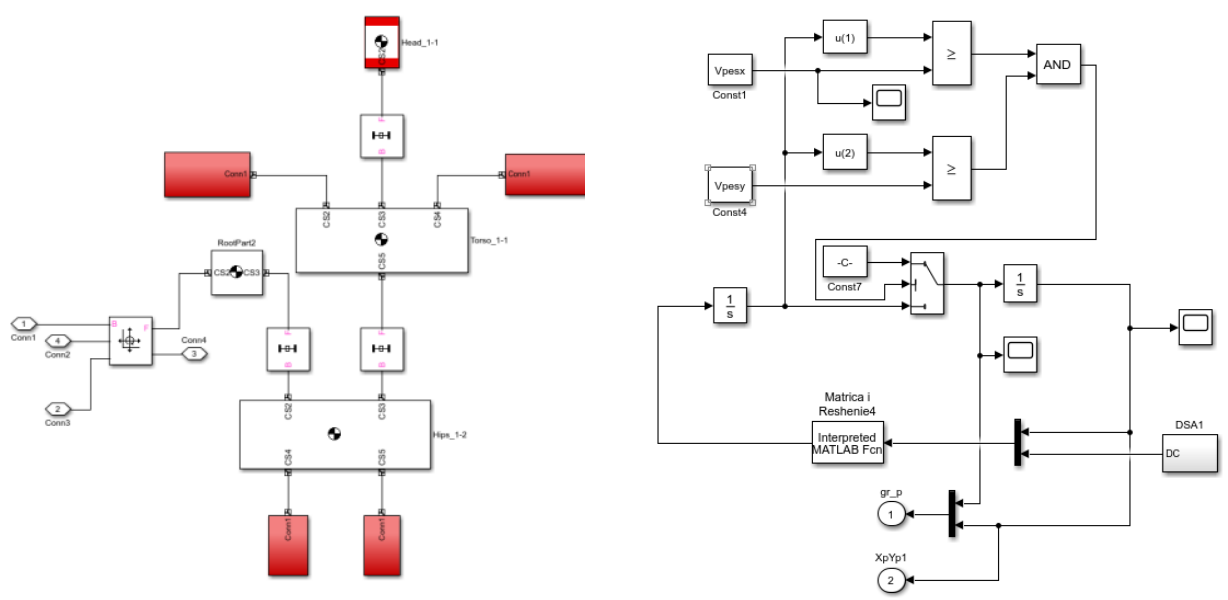

Fig. 7. Model of a human body in Matlab environment and block control 
The scheme of the control being realized of the center of mass of a person depends on types of motion - steady motion, accelerated and decelerated motion. In the system, it is possible to combine the types of motion when reaching certain indicators of the instantaneous velocity.

Fig. 8 shows the control zone in Cartesian coordinates of the center of mass of the system, as well as the angular rotation about the vertical $O z$-axis.

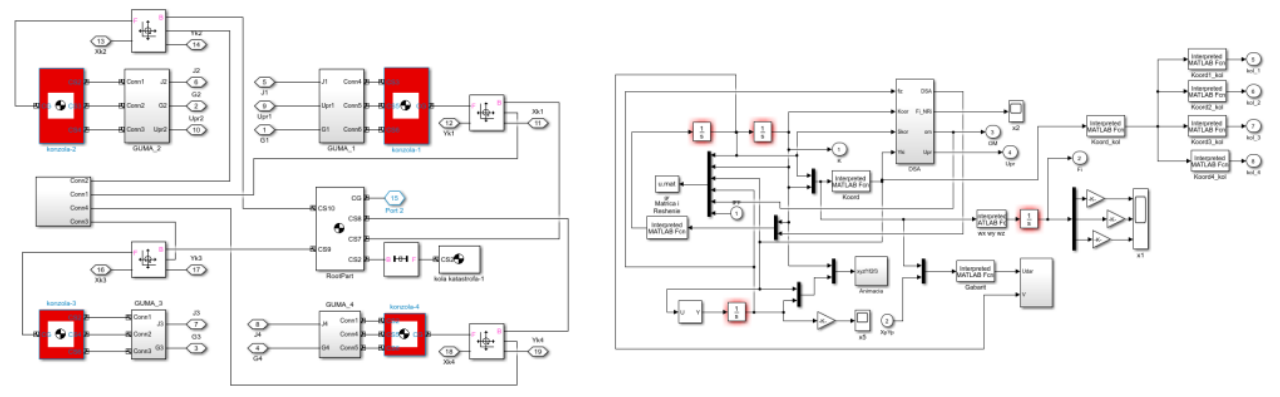

Fig. 8. Car model in Matlab environment and block-diagram of the control scheme

The proposed scheme of the realized control of the vehicle center of mass has been derived after solving the differential equations of motion for the vehicle taking into consideration the elastic characteristics of suspension, damping, and tire-road friction forces. The system of differential equations has 12 degrees of freedom - three linear displacements, three angular rotations, wheel drive and control of front-wheelsteering.

As a result, the model of spatial motion of an automobile with 12 degrees of freedom has been created.

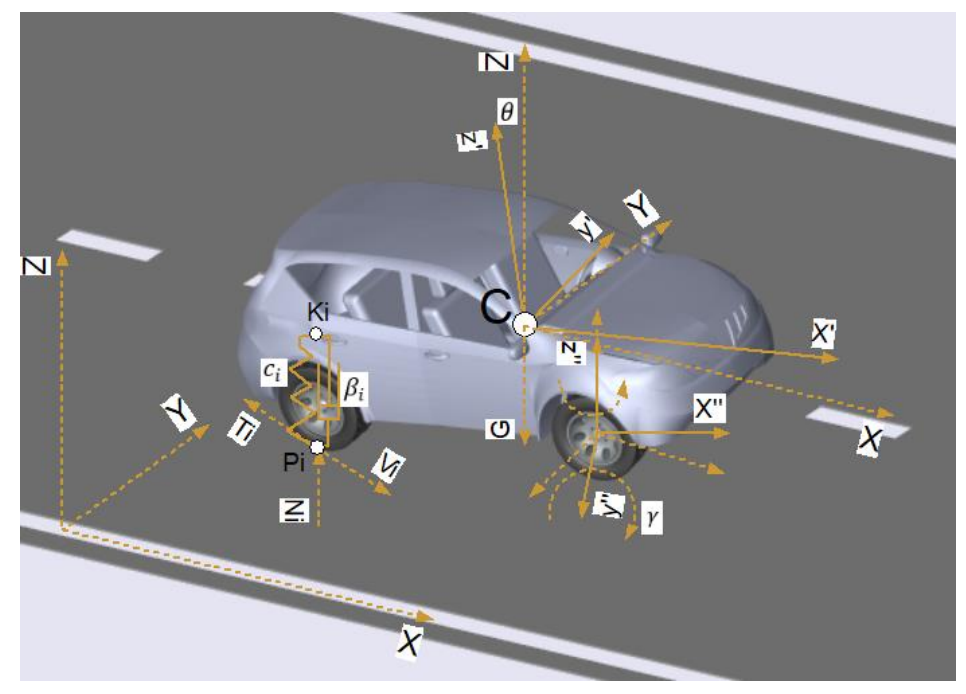

Fig. 9. Dynamic vehicle model 
The differential equations of motion of the vehicle have the form of:

$$
\begin{gathered}
m \cdot \ddot{x}=\left[\sum_{i=1}^{4} F_{x i}\right] ; m \cdot \ddot{y}=\left[\sum_{i=1}^{4} F_{y i}\right] ; \\
m \cdot \ddot{z}=\left[-G+\sum_{i=1}^{4} N_{i}-\sum_{i=1}^{4} R_{i}\right] ; \\
a_{11} \cdot \ddot{\varphi}+a_{12} \cdot \ddot{\psi}+a_{13} \cdot \ddot{\theta}= \\
=\sum_{i=1}^{4} N_{i} \cdot A F_{i}+\sum_{i=1}^{4}\left(F_{x i} \cdot f_{\varphi_{x}}+F_{y i} \cdot f_{\varphi_{y}}\right)-\sum_{i=1}^{4} R_{i} \cdot A F_{i}- \\
-b_{11} \cdot \dot{\varphi}^{2}-b_{12} \cdot \dot{\psi}^{2}-b_{13} \cdot \dot{\theta}^{2}-c_{11} \cdot \dot{\varphi} \cdot \dot{\psi}-c_{12} \cdot \dot{\varphi} \cdot \dot{\theta}-c_{13} \cdot \dot{\psi} \cdot \dot{\theta} ; \\
a_{21} \cdot \ddot{\varphi}+a_{22} \cdot \ddot{\psi}+a_{23} \cdot \ddot{\theta}= \\
=\sum_{i=1}^{4}\left(F_{x i} \cdot f_{\psi_{x}}+F_{y i} \cdot f_{\psi_{y}}\right)-b_{21} \cdot \dot{\varphi}^{2}-b_{22} \cdot \dot{\psi}^{2}-b_{23} \cdot \dot{\theta}^{2}- \\
-c_{21} \cdot \dot{\varphi} \cdot \dot{\psi}-c_{22} \cdot \dot{\varphi} \cdot \dot{\theta}-c_{23} \cdot \dot{\psi} \cdot \dot{\theta} ; \\
a_{31} \cdot \ddot{\varphi}+a_{32} \cdot \ddot{\psi}+a_{33} \cdot \ddot{\theta}= \\
=\sum_{i=1}^{4} N_{i} \cdot A T_{i}+\sum_{i=i}^{4}\left(F_{x i} \cdot f_{\theta_{x}}+F_{y i} \cdot f_{\theta_{y}}\right)-\sum_{i=1}^{4} R_{i} \cdot A T_{i}- \\
-b_{31} \cdot \dot{\varphi}^{2}-b_{32} \cdot \dot{\psi}^{2}-b_{33} \cdot \dot{\theta}^{2}-c_{31} \cdot \dot{\varphi} \cdot \dot{\psi}-c_{32} \cdot \dot{\varphi} \cdot \dot{\theta}-c_{33} \cdot \dot{\psi} \cdot \dot{\theta} .
\end{gathered}
$$

Here: $F_{i}(i=1, \ldots, 4)$ is the elastic force generated by the elasticity of car tires and springs; $N_{i}(i=1, \ldots, 4)$ is a normal reaction at the contact point of car tires, corresponding to the elastic force; $V_{i}(i=1, \ldots, 4)$ is the velocity of the contact point $P_{i}$ in the plane of the path $O x y ; T_{i}(i=1, \ldots, 4)$ is the friction force at the contact points, which lie in the plane of the path $O x y ; m$ - mass of the car; $a_{i j} ; b_{i j} ; c_{i j}$ coefficients depending on the moments of inertia; $\ddot{x}, \ddot{y}, \ddot{z}-$ acceleration of the centers of mass; $\ddot{\psi}, \ddot{\theta}, \ddot{\varphi}$ - angular acceleration by Euler angles; $\dot{\psi}, \dot{\theta}, \dot{\varphi}-$ angular velocity by Euler angles.

The solution of the equations is in matrix form and has the type of

$$
[Q]=[A]^{-1} \cdot[F],
$$

where $[Q]$ is a column matrix of the unknown linear and angular acceleration of the centers of mass of the vehicles after impact; $[A]^{-1}$ - square matrix of coefficients in front of the unknowns; $[F]$ - column matrix of generalized forces.

The relative motion of the wheels, the differential(s) and the engine is related to a system of four differential equations derived by the method of Lagrange multipliers, which has the form of

$$
\begin{gathered}
{\left[I_{\gamma}\right] \cdot[\ddot{\gamma}]=\left[M_{\gamma}\right],} \\
M_{\gamma i}=\left\{F_{i \tau} \cdot r_{i}+\operatorname{sign}\left(\dot{\gamma}_{i}\right) \cdot\left[M_{d i}-f_{i} \cdot N_{i}-M_{s i}\right]\right\},
\end{gathered}
$$

where $\vec{F}_{i \tau}$ is the tangential component of the friction force between the car tire and the road surface, the positive direction of which is taken backwards, in the more frequent case of movement when stopping or after loss of stability,

$$
F_{i \tau}=\mu \cdot N_{i} \cdot\left[\frac{V_{P i x}}{V_{P i}} \cdot \cos \left(\varphi_{z}\right)+\frac{V_{P i y}}{V_{P i}} \cdot \sin \left(\varphi_{z}\right)\right],
$$


$\mu\left(V_{P}\right)$ is the friction coefficient depending on the sliding speed of the contact spot; $\vec{r}_{i}$ - radius of the wheel; $f_{i}$-the rolling friction coefficient; $\vec{N}_{i}$ - normal reaction in the wheel; $\left[I_{\gamma}\right]$ - square matrix of the coefficients in front of the angular acceleration of the motor wheels, depending on the inertial moments of the wheels and the engine; $[\ddot{\gamma}]$ - matrix-column of the angular acceleration of the wheels with two or four propulsion system; $M_{d i}$-engine torque; $M_{s i}$ - braking torque.

The output values of the generalized coordinates of the control module represent the input values of the object created and imported from SolidWorks into Matlab [13, 18]. Motion animation is related to a real computational process of differential equations of motion.

\section{Vehicle - pedestrian collision model}

Expertise experience shows that solving the problem of pedestrian-vehicle collision is associated with several main stages of the calculation procedure - determining the velocity of the vehicle center of mass, determining the velocity of the pedestrian center of mass, investigating the possibility of preventing accidents at certain speed of motion as well as at maximum speed allowed for the road section. Apart from that, different research hypotheses are sometimes introduced, such as whether it would have been possible for the accident to be prevented by some driver actions and what actions. These hypotheses require complex computational procedures, which are sometimes not within the capabilities of real computation but of conjecture.

In the analysis shown, these various hypotheses could be expressed by setting initial conditions of motion, namely to specify the initial velocity of the center of mass, the law of change of the heading angle, the investigation of driver reaction time, etc.

In case a hazard is posed to the driver or unanticipated event places the onus upon him, he shall take action to prevent the impact. This is mainly related to the activation of the braking system and/or turning the steering wheel. Until maximum braking efficiency is reached, the total time of stopping the vehicle is driver reaction time, brake activation time and travel time delay increase. It is considered that the car travels a distance of $L$ in steady motion and it is obtained according to the dependence [7-12]:

$$
L=\left(t_{\mathrm{r}}+t_{\mathrm{sp}}+t_{\mathrm{n}}\right) \cdot V
$$

where $t_{\mathrm{r}}(\mathrm{s})$ - driver response time; $t_{\mathrm{sp}}(\mathrm{s})$ - brake activation time; $t_{\mathrm{n}}(\mathrm{s})-$ maximum travel time delay increase; $V(\mathrm{~m} / \mathrm{s})$ - pre-impact vehicle velocity of the center of mass.

The distance the car driver perceives the crash risk is obtained according to the dependence:

$$
L=\left(t_{\mathrm{r}}+t_{\mathrm{sp}}+t_{\mathrm{n}}\right) V+\frac{V_{\mathrm{bl}}^{2}-V_{y}^{2}}{2 j}
$$

where $V_{\mathrm{bl}}(\mathrm{m} / \mathrm{s})$ is the vehicle velocity of the center of mass reaching maximum efficiency; $V_{y}(\mathrm{~m} / \mathrm{s})$ is the vehicle velocity of the center of mass at the moment of impact; $j\left(\mathrm{~m} / \mathrm{s}^{2}\right)$ is the negative acceleration. 
The characteristics of the engine at forced idling or after perceiving the crash risk and the braking torque have the type, shown in Fig. 10.
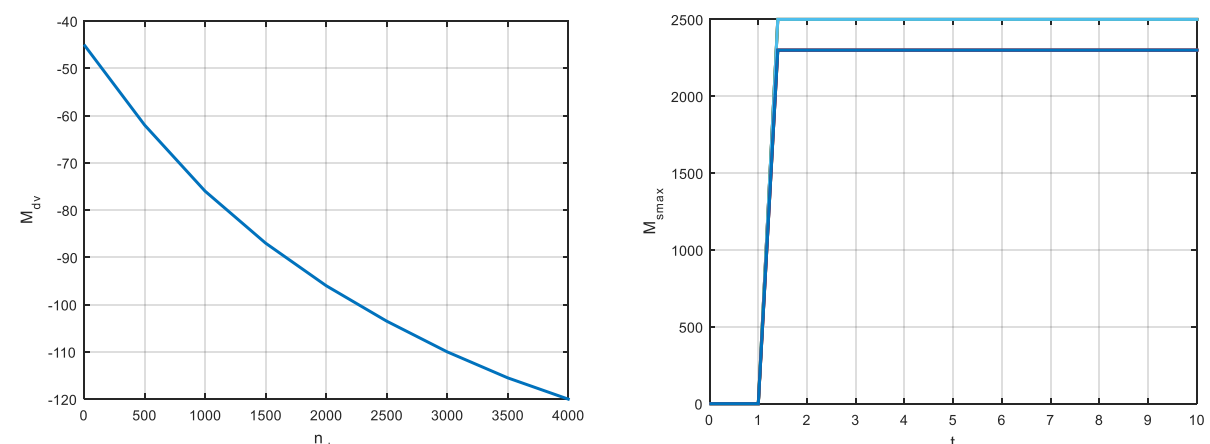

Fig. 10. Change of torque based on the RPM $n_{E}$

On the other hand, it is generally assumed that the steady motion of the pedestrian is according to the dependence

$$
L_{\mathrm{p}}=V_{\mathrm{p}} \cdot t_{\mathrm{p}}
$$

where: $V_{\mathrm{p}}$ is the pedestrian velocity of the center of mass; $t_{\mathrm{p}}$ - the pedestrian time of motion; $a_{\mathrm{p}}$ - the acceleration at the start of the pedestrian.

Actually, it is common practice the study of this motion to be associated with mixed motion in initial acceleration according to the dependence:

$$
L_{\mathrm{p}}=\frac{a_{\mathrm{p}} \cdot t_{\mathrm{p}}^{2}}{2}+V_{\mathrm{o}_{\mathrm{p}}} \cdot t_{\mathrm{p}}
$$

where $a_{\mathrm{p}}$ is the pedestrian average acceleration, $V_{\mathrm{o}_{\mathrm{p}}}$ - the initial speed of the pedestrian when entering the lane.

This computational procedure requires averaging approach to be implemented so as not to deal with dynamically changing values.

\section{Experiments to prove the operability and adequacy of the created models}

In the course of present study, experiments have been conducted with input data describing a situation of a road hazard accident with a pedestrian. Subsequently, the created model has been subjected to three variants of driver reaction in order to prevent the accident. The experiments have been performed in Matlab-Simulink environment, and each of the three variants of driver reaction has been a set of data specified by a program in the management and control units of the model designed in Matlab.

To conduct the experiments, the following activities have been carried out implementing the systems engineering information method described above:

- Geometric 3D models of a vehicle and a pedestrian were created in SolidWorks product development environment; 
- The created geometric models were exported in Matlab-Simulink environment [6].

Input data: A car moves on a straight section of a road at velocity of center of mass $V=50 \mathrm{~km} / \mathrm{h}$. A pedestrian enters on the right along the lateral direction in initial accelerated motion and at initial speed of $2 \mathrm{~km} / \mathrm{h}$. After reaching the velocity of $6 \mathrm{~km} / \mathrm{h}$ of the center of mass, the latter remains constant until the point of impact.

Variant 1. A car moves on a straight section of a road at velocity of center of mass $V=50 \mathrm{~km} / \mathrm{h}$. A pedestrian enters on the right along the lateral direction in initial accelerated motion and at initial speed of $2 \mathrm{~km} / \mathrm{h}$. After reaching the velocity of $6 \mathrm{~km} / \mathrm{h}$ of the center of mass, the latter remains constant until the point of impact.

The driver reaction time is $t_{\mathrm{r}}=0.8 \mathrm{~s}$; braking system time to collision is $t_{\mathrm{sp}}=0.2 \mathrm{~s}$; brake application delay time is $t_{\mathrm{n}}=0.4 \mathrm{~s}$; the pedestrian enters across the lane and into the auto-dominated corridor at a distance of $L=21.6 \mathrm{~m}$ from its front gauge.

Taking into account the resistance forces between the tires and the road surface, the forced idling of the engine and real-time braking torque change, the following graphical dependences are obtained (Fig. 11a and b). Fig. 11a shows graphical dependences of the change of the velocity projections of the vehicle center of mass $V_{x}$ and $V_{y}$ as well as the change of the angular velocity around the vertical axis $\omega_{z}$ as a function of the time $t$.

Fig. $11 \mathrm{~b}$ shows graphical dependences of the change in the walking speed of the pedestrian center of mass, denoted as $V_{x p}$ and $V_{y p}$, as well as the angular velocity about the vertical axis $\omega_{z p}$.

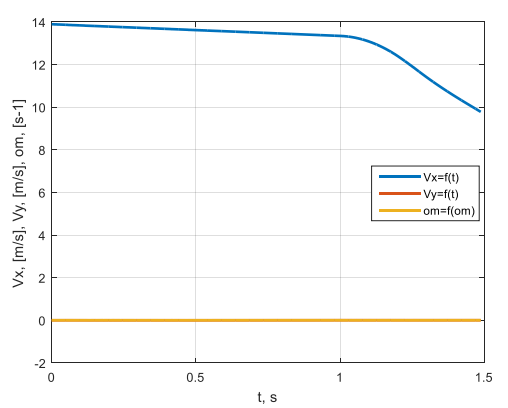

a

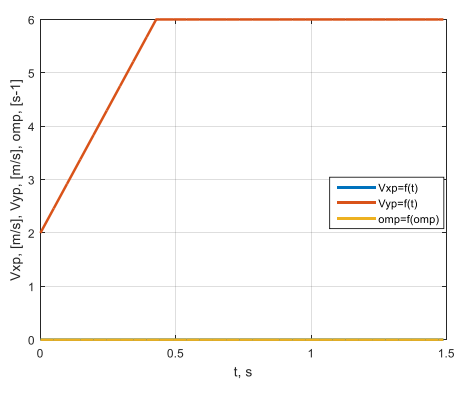

b

Fig. 11. Change of vehicle velocity center of mass and pedestrian velocity center of mass

Fig. 12a shows graphical dependences of the change of the trajectories of the center of mass of the car $x_{\mathrm{a}}=f\left(y_{\mathrm{a}}\right)$, as well as the change of the trajectories of the pedestrian center of mass $x_{\mathrm{p}}=f\left(y_{\mathrm{p}}\right)$. Fig. 12b shows graphical dependences, reflecting the change of the angular velocity of the wheels as a function of the time $t$.

Fig. 13 shows a visualization of the trajectory of both the car and the pedestrian, reflected in discrete positions of the two objects in motion to the contact area, generated in the simulation process. The solutions of the system of twelve differential equations of motion determine the change of the generalized coordinates as a function 
of time for the car at a selected time step of $0.4 \mathrm{~s}$. The solutions of the system of three differential equations of motion for the pedestrian determine the change of the generalized coordinates as a function of time at a selected integration time step of $0.4 \mathrm{~s}$. The figures show some of the position based visualisation and management of the objects.

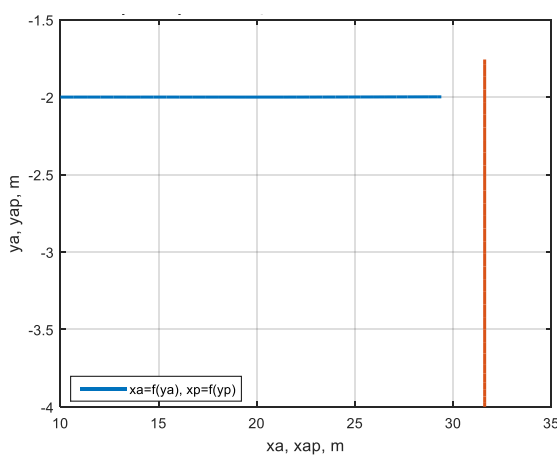

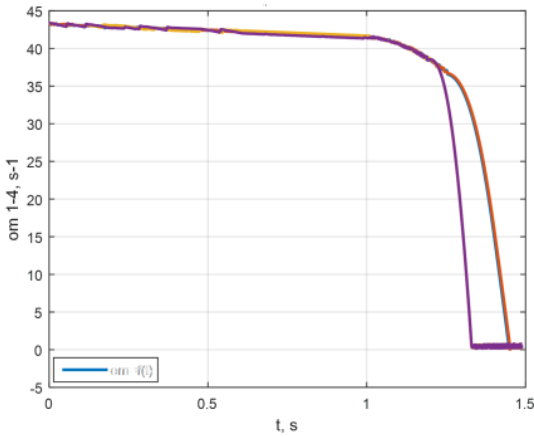

$\mathrm{b}$

Fig. 12. Trajectory of the center of mass of the car and of the pedestrian and change of the angular wheel speed

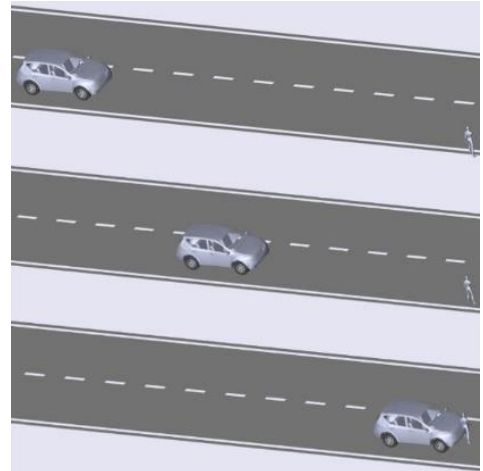

Fig. 13. Discrete event simulation model of the two objects in motion towards the point of impact

According to the dependence (8) the car speed at the moment of impact is:

$$
\begin{gathered}
V_{y}=\sqrt{V_{50}^{2}+2 j V_{50}\left(t_{\mathrm{r}}+t_{\mathrm{sp}}+0.5 t_{\mathrm{n}}\right)-2 L j}= \\
=\sqrt{13.9^{2}+2 \times 7.8 \times 13.9(0.8+0.2+0.5 \times 0.4)-2 \times 21.6 \times 7.8}= \\
=10.8 \mathrm{~m} / \mathrm{s}=38.9 \mathrm{~km} / \mathrm{h}
\end{gathered}
$$

when comparing the value according to the method, the car velocity of center of mass is $V_{y}=9.6 \mathrm{~m} / \mathrm{s}=34.6 \mathrm{~km} / \mathrm{h}$.

Based on the conducted kinematic and dynamic study, a significantly more accurate analysis of the mutual positions of the two objects in discrete positions is observed.

Variant 2. The car driver takes action to prevent the accident by turning the steering wheel. Given the same initial conditions of the two objects in motion, the 
question is as follows: What would be the mechanism of the accident and would it be possible to be prevented?

To study the motion, it is accepted that the driver turns the steering wheel left and then right with full control and without loss of lateral stability. The change of the angle of the steered wheels is shown in Fig. 14.

Taking into consideration the tire-pavement friction, the forced engine idle speed, the change of the braking torque in time, the graphical dependences are obtained. (Fig. 15). Fig. 15a shows graphical dependences of the change of the velocity projections of the vehicle center of mass $V_{x}$ and $V_{y}$, as well as the change of the angular velocity around the vertical axis $\omega_{z}$ as a function of the time $t$.

Fig. 15b shows graphical dependences of the change in the velocity of the pedestrian center of mass, denoted as $V_{x p}$ and $V_{y p}$, as well as the angular velocity about the vertical axis $\omega_{z p}$.

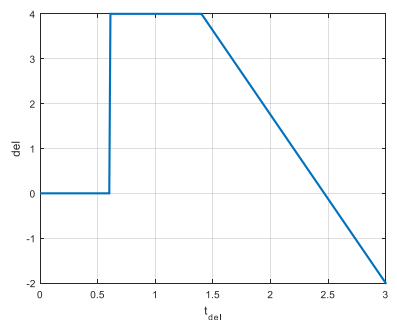

Fig. 14. Steering wheel angle of steerable wheels

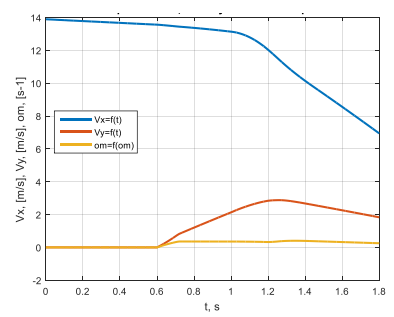

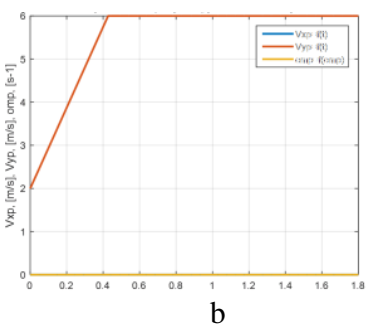

Fig. 15. Changes in the centers of mass of the car and of the pedestrian

Fig. 16a shows graphical dependences of the change of the trajectories of the vehicle center of mass $x_{\mathrm{a}}=f\left(y_{\mathrm{a}}\right)$, as well as the change of the trajectories of the pedestrian center of mass $x_{\mathrm{p}}=f\left(y_{\mathrm{p}}\right)$. Fig. 16b shows graphical dependences, reflecting the change of the angular speed of the wheels as a function of the time $t$.

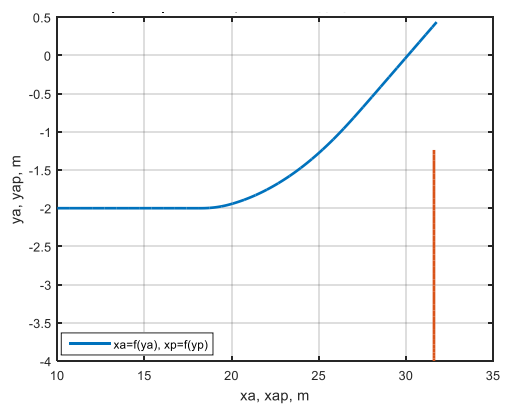

a

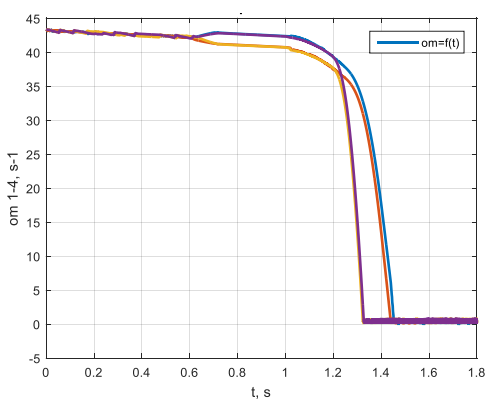

b

Fig. 16. Trajectories of the vehicle center of mass and the pedestrian center of mass. Change of the angular velocity of the car wheels

Kinematic analysis shows that the driver prevents a head-on collision with the pedestrian, but there is a side impact to the car's body. Similarly, the kinematic values can be obtained at that moment according to the undertaken actions. 
Fig. 17 shows a visualization of the car trajectory and that of the pedestrian, reflected in discrete positions of the two objects in motion to the contact area, generated in simulation process. The solutions of the system of twelve differential equations of motion determine the change of the generalized coordinates as a function of time for the car at a selected time step of $0.4 \mathrm{~s}$. The solutions of the system of three differential equations of motion for the pedestrian determine the change of the generalized coordinates as a function of time at a selected integration time step of $0.4 \mathrm{~s}$. The figures show some of the position based visualisation and management of the objects.

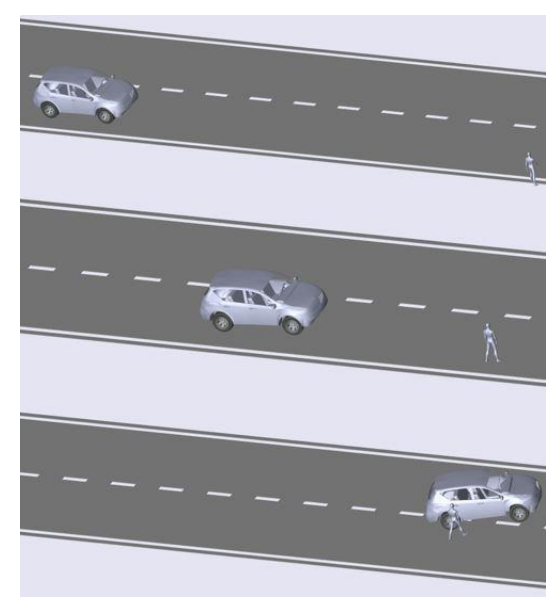

Fig. 17. Discrete event simulation model of the two objects in motion towards the point of impact

Variant 3. A car driver makes a stop while maintaining rectilinear motion and a pedestrian enters at a higher initial velocity of the center of mass of $5 \mathrm{~km} / \mathrm{h}$ and reaches an instantaneous velocity of $10 \mathrm{~km} / \mathrm{h}$.

Taking into consideration the tire-pavement friction, the forced engine idle speed, the change of the braking torque in time, the following graphical dependences are obtained (Fig. 18). Fig. 19a shows graphical dependences of the change of the velocity projections of the vehicle center of mass $V_{x}$ and $V_{y}$, as well as the change of the angular velocity around the vertical axis $\omega_{z}$ as a function of the time $t$.

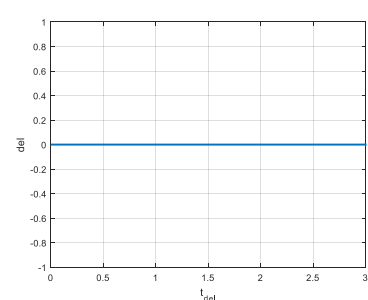

Fig. 18. Steering angle of the steering wheels

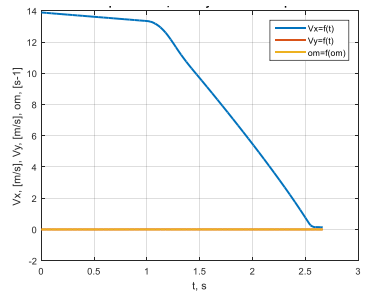

a

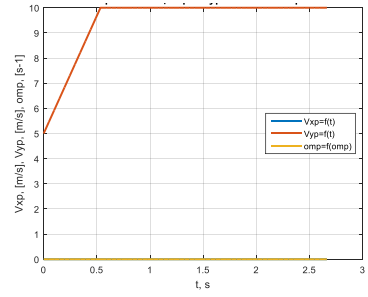

$\mathrm{b}$
Fig. 19. Velocity change of the vehicle center of mass and the pedestrian center of mass 
Fig. 19b shows graphical dependences of the change in the speed of the pedestrian center of mass, denoted as $V_{x p}$ and $V_{y p}$, as well as the angular velocity around the vertical axis $\omega_{z p}$.

Fig. 20a shows graphical dependences of the change of the trajectories of the vehicle center of mass $x_{\mathrm{a}}=f\left(y_{\mathrm{a}}\right)$, as well as the change of the trajectories of the pedestrian center of mass $x_{\mathrm{p}}=f\left(y_{\mathrm{p}}\right)$. Fig. 20b shows graphical dependences reflecting the change of the angular velocity of the wheels as a function of time $t$.

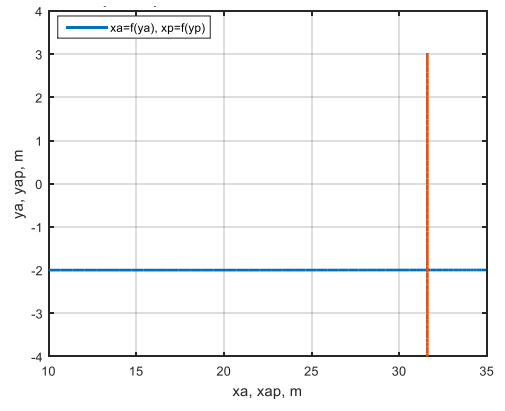

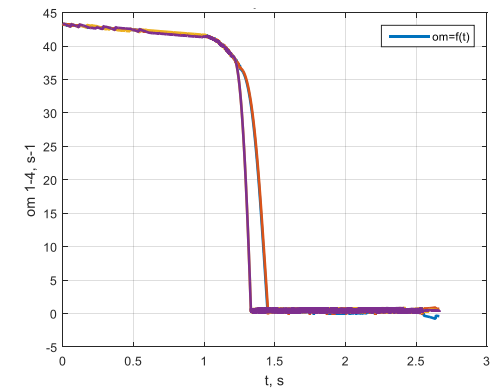

b

Fig. 20. Trajectories of the center of mass of the car and of the pedestrian center of mass and change of the angular wheel speed

Fig. 21 shows a visualization of the trajectory of the vehicle and the pedestrian, reflected in discrete positions of the two objects in motion to the place of impact, generated in simulation process. The solutions of the system of twelve differential equations of motion determine the change of the generalized coordinates as a function of time for the car at a selected time step of $0.4 \mathrm{~s}$. The solutions of the system of three differential equations of motion for the pedestrian determine the change of the generalized coordinates as a function of time at a selected integration time step of $0.4 \mathrm{~s}$. The figures show some of the position based visualisation and management of the objects.

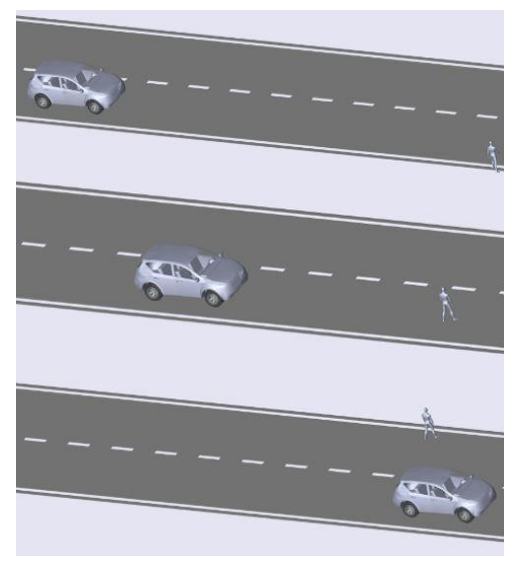

Fig. 21. Discrete event simulation model of the two objects in motion towards the point of impact 
The kinematic analysis shows that the two objects would diverge and there would be no contact between them. The car in question is at rest, and the pedestrian is outside the auto-dominated corridor.

\section{Conclusion}

The numerical experiments performed show that the proposed information model adequately reconstructs real life road traffic accidents. The designed complex model makes it possible to reconstruct various possible actions of the driver by changing the input data in the computing part. This in turn, defines the model as applicable to the study of various cases of vehicle-pedestrian collisions.

The implemented approach allows using iterations to predict with a high degree of accuracy the final outcomes and gives an answer to the frequently asked question: "What driving skills does a driver need so as to avoid a collision with a pedestrian?" generating different hypotheses of actions taken. The geometric interpretation of a traffic accident through simulation allows for accurate visualization of the behaviour and condition of objects, their geometric locations relative to each other, before, during and after the accident.

Solving the differential equations of motion of the car taking into account friction forces, elastic suspension of the suspension and tires, damping forces, gravity, dynamic characteristics of the engine at forced idling allows to predict with great accuracy the mutual positions of individual objects in motion to the point of impact. Precision is of great importance when options are small and distances short to prevent contact.

Last but not least, the realization of the proposed dynamic model with particular visualization is widely used in identification of such traffic accidents in courts.

\section{References}

1. B olkova, V., N. A. De n i s ov. System Theory and System Analysis. Moscow, Urait, 2014 (in Russian). ISBN: 987-5-9916-4213-2.

2. B o n n e t, M. Anatomy of the Collision. 2nd Edition. Jacksonville, Institute of Police Technology and Management, 2006. ISBN 1-884566-62-6.

3. Ch a r le s, V., M. B u sh e 11. Pedestrian Crash Trends and Potential Countermeasures from around the World. - Accident Anal Prev., Vol. 44, 2012, pp. 3-11. DOI: 10.1016/j. aap, 2010.

4. D u b o i s, G. Modeling and Simulation Challenges and Best Practices for Industry-Challenges and Best Practices for Industry. 2018. ISBN 9781351241137.

5. Ev s t r o p o v, M. Main Characteristics of the System from the Standpoint of a Systems Approach. Moscow, Modern Science, 2020 (in Russian). ISSN: 2414-9918.

6. J a ng, R., N. Gulle y. The Fuzzy Logic Toolbox for Use with MATLAB. Natick, MA, The MathWorks, Inc., 1995.

7. K a r a p e t k o v, S. Auto Technical Expertise. Sofia, Bulgaria, Technical University, 2005.

8. K a r a p e t k o v, S. Movement of a Car on a Plane in an Emergency Stop. Mechanics of Machines, Book 15, Varna, 2014, pp. 45-49. ISSN 0861-9727.

9. Karapetkov, S., L. Di mitrov, H. Uzunov, S. Dechkova. Identifying Vehicle and Collision Impact by Applying the Principle of Conservation of Mechanical Energy. Transport and Telecommunication, Riga, Latvia, Vol. 20, 2019, No 3, pp. 191-204. DOI: $10.2478 / \mathrm{ttj}-2019-0016$. 
10. Karapetkov, S., L. Dimitrov, H. Uzunov, S. De chkova. Examination of Vehicle Impact against Stationary Roadside Objects. - In: 9th International Scientific Conference on Research and Development of Mechanical Elements and Systems, IRMES 2019, University of Kragujevac, Faculty of EngineeringKragujevac, Serbia, 5-7 September 2019, Code 154497, Kragujevac, Serbia 2019.

11. L y u b e n o v, D., V. M a t e e v a n d, G. K a d i k y a n o v. An Expert System for Vehicle Accident Reconstruction. - EKO Varna 2019. IOP Conf. Series: Materials Science and Engineering, 614, 2019, 012006. DOI:10.1088/1757-899X/614/1/012006.

12. L y u b e n o v, D., F. K i rilov. A Study of the Influence of Input Parameters on Output Results for the Delta V Method. - Proceedings of University of Ruse, Vol. 58, 2019, Book 4, pp. 170-176. ISSN 1311-3321 (print); ISSN 2603-4123 (on-line).

13. MathWorks. MATLAB, Realise $2019 \mathrm{~b}$.

14. Mingyue, M., Y. Yanqun, Y. Li ang. Pedestrian Crash Risk Assessment and Intervention. - Advances in Mechanical Engineering, Vol. 8, 2016, No 7, pp. 1-10. DOI: $10.1177 / 1687814016653296$.

15. Ni e h of f, P., C. G a b l e r. The Accuracy of WinSMASH Delta-V Estimates: The Influence of Vehicle Type, Stiffness, and Impact Mode. - In: Proc. of 50th Annual Association for the Advancement of Automotive Medicine, Chicago, IL, 2006.

16. R e a d, M., P. S a $1 \mathrm{~m}$ o n, M. L e $n \mathrm{n}$ e, N. S t a $\mathrm{n}$ t o n. Walking the Line: Understanding Pedestrian Behavior and Risk at Rail Level Crossings with Cognitive Work Analysis. - Applied Ergonomics, Vol. 53, 2016, Part A, pp. 209-227. DOI:10.1016/j.apergo.2015.10.004.

17. S e th, A., R. M a ti a s, A. V e l o s o, S. D e 1 p. A Biomechanical Model of the Scapu-Lothoracic Joint to Accurately Capture Scapular Kinematics during Shoulder Movements. - PLOS ONE, Vol. 11, 2016, pp. 1-18.

18. S h a m, T. Solid Works Simulation 2018. A Tutorial Approach. Purdue University Northwest, USA, 2018.

19. S t u c k i, L., O. Fe s s a h a i e. Comparison of Measured Velocity Change in Frontal Crash Tests to NASS Computed Velocity Change. SAE 980649, Society of Automotive Engineers, 1998.

Received: 01.09.2020; Second Version: 27.01.2021; Accepted: 04.02.2021 